\title{
MINERALES ARCILLOSOS PROTOCOLO ESPECIALIZADO DE CATEGORIZACIÓN CERÁMICA: UNA REVISIÓN
}

\author{
CLAY MINERALS \\ SPECIALIZED CERAMIC CATEGORIZATION PROTOCOL:
}

A REVIEW

\section{Rafael Uribe $^{1 *}$, Francesco Camillo $^{2}$ \& Luis Lascano ${ }^{3}$}

Recibido: 10 de noviembre 2020 / Aceptado: 24 de junio 2021

DOI 10.26807/ia.v9i2.202

Palabras clave: Arcillas, caracterización cerámica, minerales arcillosos, minerales no metálicos, propiedades cerámicas.

Keywords: Clays, ceramic characterization, clay minerals, non-metallic minerals, ceramic properties.

\section{RESUMEN}

Las relaciones entre la estructura-diseño-procesamiento-propiedades de los materiales cerámicos son por lo general complejas y objeto de continuos estudios, sea el caso de predecir propiedades a partir de una estructura o lograr estructuras

1 Escuela Politécnica Nacional, Departamento de Ingeniería Química. Quito, Ecuador ( ${ }^{*}$ correspondencia: rafael.uribe@epn.edu.ec)

2 Instituto Universitario de Tecnología "Dr. Federico Rivero Palacio", Dpto. de Tecnología de Materiales, Caracas, Venezuela (francamil @yahoo.com)

3 Escuela Politécnica Nacional, Departamento de Física. Quito, Ecuador. (luis.lascano@epn.edu.ec) 
que garanticen ciertas propiedades. Así, el uso de técnicas especializadas de caracterización de materias primas y productos es fundamental para prever el potencial de uso de un lote, concertar su influencia en producción y prevenir las variaciones que generan pérdidas, minimizan la productividad y la calidad en los productos. En Latinoamérica, y en especial en el Ecuador, son desconocidas la mayoría de las técnicas especializadas de caracterización de materiales cerámicos, que parten de la evaluación de las características más importantes de los polvos y que tienen influencia significativa en los distintos procesos de fabricación de materiales y en el desarrollo de los denominados nuevos materiales. En tal sentido, en este artículo se presenta una revisión minuciosa de las técnicas especializadas de caracterización y valoración de minerales arcillosos, que servirán de guía al sector cerámico artesanal e industrial del Ecuador. Se describen las propiedades más importantes de los materiales cerámicos, a fin de introducir de manera directa a los interesados en el conocimiento de un conjunto de técnicas y métodos "convencionales" y "no convencionales" de caracterización, utilizados en la valorización especializada de materias primas, procesos y materiales cerámicos; incluidos los aspectos tecnológicos.

\section{ABSTRACT}

The relationships between the structure-design-processing-properties of ceramic materials are generally complex and the subject of continuous studies, whether it is the case of predicting properties from a structure or achieving structures that guarantee certain properties. Thus, the use of specialized techniques for the characterization of raw materials and products is essential to foresee the potential of use of a batch, coordinate its influence on production and prevent variations that generate losses, minimize productivity and quality in products. In Latin America, and especially in Ecuador, most of the specialized techniques for characterization of ceramic materials are unknown, which start from the evaluation of the most important characteristics of powders, and which have a significant influence on the different manufacturing processes of materials and in the development of so-called new materials. In this sense, this article presents a detailed review of the specialized techniques for characterization and valua- 
tion of clay minerals, which will serve as a guide to the artisanal and industrial ceramic sector of Ecuador. The most important properties of ceramic materials are described, in order to introduce directly to those interested in the knowledge of a set of "conventional" and "unconventional" characterization techniques and methods, used in the specialized recovery of raw materials ceramic materials and processes, including technological aspects.

\section{INTRODUCCIÓN}

En pleno siglo XXI, la sociedad requiere día a día de nuevos materiales con propiedades y características que le permitan satisfacer su creciente demanda de necesidades. La posibilidad de fabricar nuevos materiales con propiedades más exactas y reproducibles, se multiplican a medida que los centros de investigación científica y tecnológica van alcanzando y desarrollando técnicas y métodos más precisos de caracterización y valorización de materias primas naturales y sintéticas, preparación, obtención y conformado de materiales y, de evaluación precisa de la respuesta en servicio y uso que tendrán los materiales, es decir sus propiedades (Uribe et al., 2020).
Lo interesante de esta época, sin lugar a duda, es que cualquier característica y propiedad de un material o materia prima, puede ser contrastable en cualquier laboratorio del mundo, gracias a que se ha dado paso a la normalización mundial de métodos, ensayos y prácticas de análisis, acompañadas éstas de equipos de medición de alta tecnología, de gran precisión y exactitud (Hevia, 2012). De esta manera, el aporte actual de la ciencia e ingeniería de materiales está dado por la posibilidad no solo de caracterizar sino también de evaluar, predecir y diseñar el comportamiento de los nuevos materiales $y$, lo más importante, valorar su utilidad en diversas aplicaciones (Sánchez et al., 1998). 
Las relaciones entre la estructura-diseño-procesamiento-propiedades de los materiales, y más aún en las del denominado grupo de los nuevos materiales, son por lo general complejas, y objeto de continuos estudios, sea el caso de predecir propiedades a partir de una estructura o lograr estructuras que garanticen ciertas propiedades; sin dejar de considerarse, a su vez, que las propiedades de los productos están determinadas no solo por la composición y estructura de las fases presentes sino por el arreglo de éstas (Melgarejo et al., 2010). La distribución de las fases o microestructura del cuerpo final del producto depende de las técnicas, condiciones y procesos de fabricación, las materias primas utilizadas, y de ser el caso, las relaciones de equilibrios y cinéticas de cambios de fase, crecimientos de grano y sinterización, como ocurre en los materiales cerámicos (Díaz et al., 2002). Aspectos básicos que nos inducen al estudio de un sinfín de sistemas, de los cuales solo se puede obtener información gracias a un importante conjunto de técnicas y métodos "convencionales" y "no convencionales" de caracterización y valorización de materias primas, pro- cesos y materiales (Guillen et al., 2012).

En casi todas las industrias relacionadas con el procesamiento y fabricación de materiales metálicos, poliméricos y cerámicos, se utiliza materiales en polvo, los cuales están constituidos por partículas primarias o por aglomerados de ellas. Las partículas primarias son moléculas o cristales imperfectos que presentan una estructura de tipo mosaico, conformada a su vez por unas pocas celdas unitarias. Por ejemplo, en el caso de los materiales cerámicos, el tamaño de los monocristales es de $10^{-6}$ a $10^{-8} \mathrm{~cm}$, tamaño de cristalito. Los cristalitos se pueden organizar de manera cooperativa y sistemática generando partículas primarias monocristalinas, o simplemente estar dispersos al azar produciendo partículas primarias policristalinas (GuiIlen et al., 2012). Un aglomerado o partícula secundaria es un ensamble de partículas primarias que contiene una red de poros interconectados. En el caso de los materiales cerámicos, si los aglomerados se forman durante la síntesis del polvo cerámico, su tamaño y forma son difíciles de controlar; una situación diferente se 
presenta cuando se utilizan procesos de granulación (Fernández et al., 2003).

A la hora de seleccionar el material cerámico más adecuado para una determinada aplicación, debemos tener en cuenta diversos factores como: el trabajo que va a desarrollar la pieza, la atmósfera en la que se va a encontrar, el proceso de conformado mediante el cual se le dará la forma definitiva, la disponibilidad de ese material, su coste, el cual no debe suponer más de la mitad del precio final del producto, para que su venta pueda resultar competitiva (incluye extracción, transporte, transformación en producto de primera fase y transporte al lugar de la segunda transformación). Por lo tanto, un profundo conocimiento de las propiedades y de las formas en que se pueden mejorar es la clave para estar en condiciones de determinar cuál es el más adecuado (Villalba, 2020).

La mayoría de las técnicas de caracterización más usuales de materiales parten de la evaluación de las características más importantes de los polvos, que tienen influencia significativa en los distintos procesos de fa- bricación de materiales y en el desarrollo de los denominados nuevos materiales (Dávila et al., 2011). Se realizan evaluaciones de las características físicas (tamaño, morfología y distribución de partículas, densidad y peso específico, superficie específica, porosidad, grado de agregación, facilidad de separación por filtración, sublimación, evaporación y/o destilación, entre otras), características químicas (composición en elementos y óxidos, reactividad, estabilidad, solubilidad, entre otras ) y en la actualidad es fundamental considerar los factores ecológicos, tales como la posibilidad de reciclaje y reutilización, uso y costo mínimos de explotación y/o procesamiento (Cely et al., 2015).

Los polvos cerámicos merecen especial atención, por encontramos en la "Era del Silicio - Era de la Información" (Belmonte et al., 2010), sería imposible imaginar que ocurriría hoy en día si todos los dispositivos de Silicio dejarán de funcionar (Fernández et al., 2003; Kingery, 1976). Estos polvos presentan:

- Un valor alto de la relación área superficial por unidad de peso, superficie específica y de la energía superficial. 
- Innumerables discontinuidades en la superficie que hacen que sus propiedades superficiales (absorción, reacción y reactividad) sean más importantes que las relacionadas con la estructura interna del sólido.

- Propiedades fluctuantes de las partículas como el tamaño, morfología y distribución, que tienen gran influencia en el empaquetamiento y grado de dispersión que ellas presentan. Fenómenos que hacen su ocurrencia de naturaleza probabilística y estadística.

El conformado es la etapa del procesamiento en la que el sistema de partículas aisladas se transforma en un cuerpo consolidado con una geometría y microestructura específica. Un rasgo distintivo de los procesos cerámicos es que están basados mayoritariamente en la tecnología de polvos, dado que los altos puntos de fusión o descomposición de los materiales cerámicos impiden el empleo de técnicas de deformación, típicas de los metales (Souza et al., 2002). La selección del método de conformado depende de la cantidad, geometría y homogeneidad de las piezas y de la reproducibilidad del proceso. Esto exige el cuidadoso control y evaluación de las características fisicoquímicas del material particulado y de los aditivos necesarios para optimizar cada etapa del proceso (Fernández et al., 2003). Nuevas técnicas y métodos experimentales de evaluación de materias primas naturales y sintéticas y de nuevos materiales, han dado paso al control tecnológico sistematizado de los procesos clásicos de obtención de materiales (prensado axial e isostático, extrusión, moldeo por inyección, filtración, entre otros) y han impulsado el avance procesos no tradicionales de obtención de nuevos materiales como: deposición, evaporación, coagulación y gelificación, intercalación, deposición física o química de películas delgadas a partir de la fase vapor, "physical vapour deposition" o PVD y "chemical vapour deposition” o CVD (Dávila et al., 2011).

Dada la gran variedad en su composición y en su estructura los materiales cerámicos, presentan una amplitud notable en los valores de sus propiedades. Por lo cual, gracias a que el empirismo sin dirección tecnoló- 
gica ha disminuido considerablemente desde finales del siglo XX, podemos afirmar que en la actualidad se tiene el control de las propiedades. La experiencia ha demostrado que el enfocar la atención en la relación estructura-diseño-procesamiento-propiedades ha sido un poderoso $y$ efectivo tratamiento.

En tal sentido, en este artículo se presenta un conjunto de técnicas especializadas de caracterización y valoración de minerales arcillosos, que servirán de guía al sector cerámico artesanal e industrial del Ecuador. Se describen las propiedades más importantes de los materiales cerámicos, a fin de introducir al lector de manera directa en el conocimiento de un conjunto de métodos "convencionales" y "no convencionales" de caracterización, utilizados en la valorización especializada de materias primas, procesos y materiales cerámicos; incluidos los aspectos tecnológicos.

\section{MATERIALES Y MÉTODOS}

\section{Métodos de estudio de afloramientos minerales no metálicos}

El estudio que se realiza para la valoración de un afloramiento mineral con frecuencia implica tan sólo el estudio genérico de los elementos que influyen en la comercialización del depósito, obviándose en muchos casos el estudio mineralógico detallado de las propiedades de los minerales (García et al., 1985). No obstante, un estudio detallado de la mineralogía del afloramiento no tiene tan sólo connotaciones científicas, como a veces se cree, sino que ayuda a incre- mentar el valor añadido de la explotación, e incluso por sí solo puede ayudar a descartar o confirmar el interés del yacimiento (Torres et al., 2011).

En su trabajo, Uribe et al. (2020) evidenciaron que en Latinoamérica, y en especial en el Ecuador, es escasa la información que se tiene sobre las propiedades tecnológicas de las materias primas utilizadas en el sector cerámico. Pocos industriales, artesanos y centros de investigación realizan estudios formales para minimizar y controlar el efecto de las materias 
primas en la productividad. Estos investigadores, ofrecieron una revisión de las técnicas básicas de caracterización mineral más usuales. El protocolo revisado permite obtener datos importantes para evaluar la factibilidad de uso de las arcillas, como potenciales materias primas dentro de composiciones de pastas para la elaboración artesanal/industrial de productos cerámicos.

El peso del factor materia prima en las pérdidas de calidad de los productos, evidencia el carácter obligatorio de políticas gerenciales y el uso de técnicas apropiadas, tales como el control de la variabilidad de la mina, la homogenización de la materia prima y/o el ajuste oportuno de los parámetros de trabajo en el proceso productivo. Es importante conocer las características y pautas de preparación de las muestras a ensayar, para reducir el riesgo de cometer errores en los ensayos y no obtener resultados representativos de las características y propiedades del lote a ensayar.

En el caso de la caracterización fisicoquímica y cerámica de minerales arcillosos tiene como prioridad cono- cer las aplicaciones prácticas y potencialidades de uso, solas o combinadas en pastas cerámicas de las materias primas en la industria cerámica. Este estudio incluye: el control por atributos, tanto cualitativo como cuantitativo, el estudio de factibilidad de uso cerámico artesanal y/o industrial y, la caracterización cerámica especializada de minerales arcillosos (Uribe et al., 2020).

El procesamiento de un material cerámico implica que las características estructurales y constitucionales de los materiales de partida se van desarroIlando y modificando en cada estadio sucesivo que conlleva el procesamiento. Si se representa el procesamiento como una serie de operadores, el producto final contendrá términos que representan todas las operaciones del proceso, así como las características de los materiales de partida (Martín et al., 1977). Por lo tanto, cualquier cambio en las características de los materiales de partida o en cualquier operación del procesamiento producirá cambios en las características del producto final. Una vez que se evidencian con la evaluación anterior las propiedades básicas de los minerales estudiados y 
en lo específico se observa un comportamiento plástico (arcillosos) de la muestra de mineral, la misma es sometida a distintos procesos de evaluación cerámica especializada a fin de determinar su factibilidad de uso cerámico artesanal y/o industrial (Hevia, 2012). Por lo general la muestra de mineral arcilloso es sometida a:

- Tomas de muestras para realización de ensayos especializados, de acuerdo con los requerimientos de cada norma o protocolo de ensayo específico.

- Proceso de beneficiado (trituración y molienda).

- Proceso de preparación de pasta (seca granulada, plástica o barbotina).

- Elaboración de probetas de ensayo (prensado, extrusión, vaciado).
- Proceso de secado de probetas y/o muestras a $120{ }^{\circ} \mathrm{C}$, durante 12 horas o hasta obtener un peso constante.

- Proceso de cocción bajo ciclos controlados, en hornos eléctricos de laboratorio, con un mínimo de permanencia de media hora a la temperatura máxima de cocción. Las muestras se someten a distintos ciclos de cocción, a temperaturas máximas diferentes entre los 850 y $1200{ }^{\circ} \mathrm{C}$, con el objeto de determinar el intervalo de cocción más adecuado para el tipo de mineral arcilloso (Espinoza et al., 1978). En la Tabla 1 se presenta una lista de las normas ASTM que delinean los procedimientos normalizados de caracterización de materias primas y productos cerámicos.

Tabla 1. Listado de normas de caracterización cerámica especializada

Norma

ASTM C322

Standard Practice for Sampling Ceramic Whiteware Clays. (Práctica estándar para el muestreo de arcillas de cerámica blanca)

ASTM C323 Standard Test Methods for Chemical Analysis of Ceramic Whiteware Clays. (Métodos de prueba estándar para el análisis químico de arcillas de cerámica blanca)
Standard Test Method for Free Moisture in Ceramic Whiteware Clays. (Método de prueba estándar para humedad libre en arcillas de cerámica blanca)
Standard Guide for Wet Sieve Analysis of Ceramic Whiteware Clays. (Guía estándar para el análisis por tamizado húmedo de arcillas de cerámica blanca) 
ASTM C326

Standard Test Method for Drying and Firing Shrinkages of Ceramic Whiteware Clays.

(Método de prueba estándar para el secado y cocción de contracciones de arcillas de cerámica blanca)

ASTM C327 Method of Test for Linear Thermal Expansion of Fired Ceramic Whiteware Materials. (Método de prueba para la expansión térmica lineal de materiales de cerámica blanca cocidos)

ASTM C539

Standard Test Method for Linear Thermal Expansion of Porcelain Enamel and Glaze Frits and Ceramic Whiteware Materials.

(Método de prueba estándar para la expansión térmica lineal de fritas de esmalte y esmalte de porcelana y materiales de cerámica blanca)

ASTM C328 Test Methods for Test for Modulus of Rupture of Fired, Dry-Pressed Ceramic Whiteware Specimens at Normal Temperature.

(Métodos de prueba para el ensayo del módulo de ruptura de muestras de cerámica blanca cocidas y prensadas en seco a temperatura normal)

ASTM C674 Standard Test Methods for Flexural Properties of Ceramic Whiteware Materials.

(Métodos de prueba estándar para las propiedades de flexión de materiales de cerámica blanca)

ASTM C329

Standard Test Method for Specific Gravity of Fired Ceramic Whiteware Materials.

(Método de prueba estándar para la gravedad específica de materiales de cerámica blanca cocidos)

ASTM C370 Standard Test Method for Moisture Expansion of Fired Whiteware Products. (Método de prueba estándar para la expansión de la humedad de productos de cerámica cocida)

ASTM C371 Standard Test Method for Wire-Cloth Sieve Analysis of Nonplastic Ceramic Powders.

(Método de prueba estándar para el análisis por tamiz de tela metálica de polvos cerámicos no plásticos)

ASTM C372 Standard Test Method for Linear Thermal Expansion of Porcelain Enamel and Glaze Frits and Fired Ceramic Whiteware Products by the Dilatometer Method.

(Método de prueba estándar para la expansión térmica lineal de fritas de esmalte y glaseado de porcelana y productos de cerámica cocida por el método dilatómetro)

ASTM C373 Standard Test Method for Water Absorption, Bulk Density,

Apparent Porosity, and Apparent Specific Gravity of Fired Whiteware Products.

(Método de prueba estándar para absorción de agua, densidad aparente, porosidad aparente y gravedad específica aparente de productos de cerámica cocida)

ASTM D153

Test methods for specific gravity pigments.

(Métodos de prueba para pigmentos de gravedad específica.)

ASTM C501 Relative resistance to wear of unglazed ceramic tile by the taber abraser.

(Resistencia relativa al desgaste de baldosas de cerámica sin esmaltar por el abrasivo taber)

ASTM C242 Terminology of ceramic whiteware and related products. (Terminología de cerámica blanca y productos relacionados)

ASTM C554 Crazing resistance of fired glazed ceramic whiteware by a thermal shock method. (Resistencia al agrietamiento de la cerámica esmaltada cocida por métodos de choque térmico) 
Density of glass by the sink-float comparator.

(Densidad del vidrio por el comparador de flotador-fregadero).

ASTM C824

Specimen preparation for determination of linear thermal expansion of vitreous glass enamels and glass enamel frits by the dilatometer methods.

(Preparación de muestras para la determinación de la expansión térmica lineal de esmaltes de vidrio vítreo y fritas de esmalte de vidrio mediante los métodos del dilatómetro)

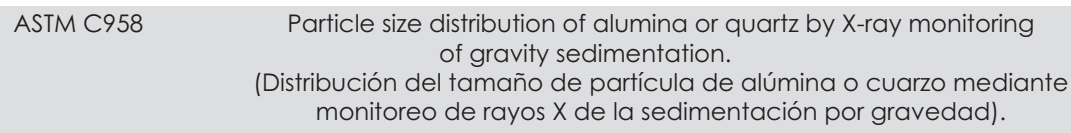

ASTM C204

Standard Test Methods for Fineness of Hydraulic Cement by

Air-Permeability Apparatus. (Métodos de prueba estándar para determinar la finura del cemento hidráulico mediante aparatos de permeabilidad al aire)

\begin{tabular}{|c|c|}
\hline ASTM C 1069 & $\begin{array}{l}\text { Specific surface area of alumina or quartz by nitrogen absorption. } \\
\text { (Superficie específica de alúmina o cuarzo por absorción de nitrógeno) }\end{array}$ \\
\hline ASTM C 1251 & $\begin{array}{c}\text { Determination of specific surface area of advanced ceramic materials } \\
\text { by gas adsorption. } \\
\text { (Determinación del área de superficie específica de materiales cerámicos } \\
\text { avanzados por adsorción de gas). }\end{array}$ \\
\hline ASTM C 1274 & $\begin{array}{l}\text { Advanced ceramic specific surface area by physical adsorption. } \\
\text { (Superficie específica de cerámica avanzada por adsorción física) }\end{array}$ \\
\hline ASTM C 1286 & $\begin{array}{l}\text { Standard classification for advanced ceramics. } \\
\text { (Clasificación estándar para cerámica avanzada). }\end{array}$ \\
\hline
\end{tabular}

Estas normas cubren los procedimientos especializados que van desde la determinación mediante protocolos sencillos de la absorción de agua, densidad aparente, porosidad aparente y gravedad específica aparente de piezas cerámicas sin esmalte, hasta los métodos para determinar los coeficientes lineales de expansión térmica o la superficie especifica de polvos, dentro de los cuales se requiere de equipos costosos de alta tecnología. La medida de estas propiedades, en conjuntos con las técnicas de análisis térmico, mineralógico y microestructural, que se discutirán en los apartados subsiguientes, conforman herramientas para la determinación del grado de maduración de un cuerpo cerámico o para la determinación de las propiedades estructurales que pueden ser requeridas para una aplicación dada. 


\section{RESULTADOS Y DISCUSIÓN}

\section{Estudio especializado de factibilidad de uso de minerales arcillosos}

\section{Medidas de las propiedades físicas}

\section{Densidad}

La composición de la corteza terrestre primitiva era uniforme en toda la superficie del globo. La variedad que caracteriza a la superficie terrestre actual ha sido la consecuencia de los cambios posteriores, inducidos por los distintos fenómenos y eras geológicas. Sin embargo, estos cambios han afectado sólo a la superficie. Los materiales más pesados, como el níquel (densidad $8,9 \mathrm{~g} / \mathrm{cm}^{3}$ ) y el hierro (densidad 7,8 g/ $\mathrm{cm}^{3}$ ), se fueron hundiendo hacia el interior de las capas más profundas; mientras que los más ligeros, como el silicio $(\mathrm{d}=2,42$ $\left.\mathrm{g} / \mathrm{cm}^{3}\right)$ y el aluminio $\left(\mathrm{d}=2,7 \mathrm{~g} / \mathrm{cm}^{3}\right)$, se fueron concentrando en las capas más superficiales (Fernández, 2010).

La densidad absoluta o masa volúmica específica de los materiales es una propiedad física que define su grado de concentración de masa, es decir, el grado de compactación o empaquetamiento de la estructura.
En los materiales cerámicos es una característica primordial para controlar tanto en la materia prima como en el producto final. Así, por ejemplo, en el caso de las barbotinas, es parámetro primordial para la obtención de suspensiones con niveles de concentración de sólidos y fluidez suficiente para ser colada (controlada esta última por el uso de defloculantes); y en otros casos el nivel de densificación de un producto terminado (densidad aparente) da una idea clara de su estado de sinterización, caracterizando el uso de los materiales como lozas, gres, refractarios, aislantes térmicos (Gómez et al., 1990). En la Figura 1 se muestra un esquema de los métodos más comunes de determinación de densidad, utilizados en función de estado de materia. Es importante acotar que el valor obtenido de la densidad de un material depende mucho del método de preparación de la muestra, por lo que es aconsejable que siempre se indique el protocolo o la norma seguida para la determinación de esta propiedad. La densidad de los feldespatos alcalinos varía entre 2,5 y 2,6 $\mathrm{g} / \mathrm{cm}^{3}$, la de los caolines varía entre 
2,4 y $2,65 \mathrm{~g} / \mathrm{cm}^{3}$, las arenas/ sílice entre 2,5 y $2,8 \mathrm{~g} / \mathrm{cm}^{3}$ y las arcillas valores promedio del orden de los 2,7 $\mathrm{g} / \mathrm{cm}^{3}$ (Uribe, 2015).

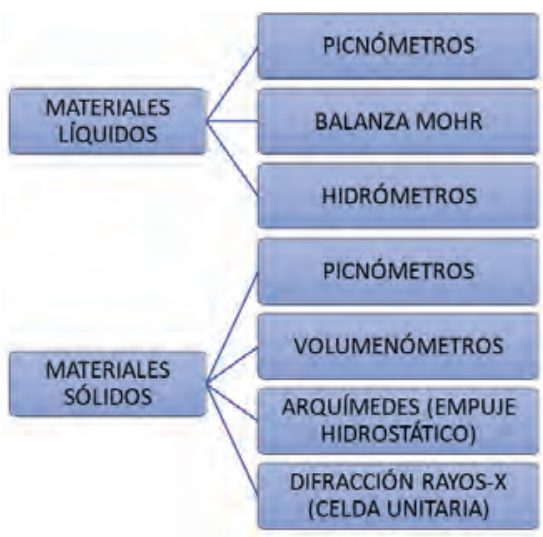

Figura 1. Esquema de métodos comunes de determinación de densidad

\section{Superficie específica}

Un problema de enorme trascendencia tanto desde el punto de vista fundamental como aplicativo es conocer la magnitud absoluta del área superficial de los sólidos, conocida como Superficie Específica $\left(\mathrm{S}_{0}\right)$ (Albella, 1999). La superficie específica de un sólido sin poros es aproximadamente igual al área superficial geométrica, pero para sólidos porosos esta superficie puede ser muy grande, la $\mathrm{S}_{0}$ se expresa en $\mathrm{cm}^{2}$ de superficie por gramo de material en polvo, o bien por unidad de volumen del cuerpo. Entre los métodos más utilizados se pueden mencionar: (Pinto, 2011)

- Métodos microscópicos: Tediosos pero preciso, no apto para control de calidad.

- Permeabilidad de un gas: Se determina la $S_{0}$ para un grado de compactación (densidad aparente) de un polvo, midiendo la variación de flujo de aire. consiste esencialmente de un mecanismo que permite pasar una cantidad definida de aire a través de una camada o pastilla preparada con una porosidad definida. El número y tamaño de los poros en una camada preparada de porosidad definida es una función del tamaño de las partículas y determina la velocidad de flujo del aire a través de la camada.

- Método BET (desarrollado por Brunauer, Emmett y Teller): Es una medida directa de la superficie específica, $\mathrm{S}_{0}$, se basa en la absorción de nitrógeno a baja temperatura $\left(-196{ }^{\circ} \mathrm{C}\right)$. Permite determinar la $\mathrm{S}_{0}$ tanto de un polvo como de los poros abiertos de un material poroso. La evaluación de la $S_{0}$ a partir de una isoterma de absorción física requiere de la determinación del vo- 
lumen de gas necesario $\left(\mathrm{V}_{\mathrm{m}}\right)$ para formar una capa molecular recubriendo la totalidad de la superficie del sólido. Por lo tanto, a partir del valor de la capacidad de la monocapa $\left(\mathrm{V}_{\mathrm{m}}\right)$ obtenido de la correspondiente isoterma de absorción, se puede calcular el valor de la $S_{0}$.

Para dar idea de valores, puede mencionarse que la superficie específica de un ladrillo refractario puede estar entre 0,2 y $1,0\left(\mathrm{~m}^{2} / \mathrm{g}\right)$, una arena para fabricar vidrio tiene entre 1,0-1,5, las arcillas dependiendo de su naturaleza mineralógica: Montmorillonitas entre 80-300, Illita hasta 50, Halloysita hasta 60, Caolinita de baja cristalinidad hasta 50 y Caolinita de elevada cristalinidad hasta $15\left(\mathrm{~m}^{2} / \mathrm{g}\right)$ (Kingery, 1976).

\section{Tamaño de grano}

El análisis granulométrico de un producto consiste en conocer las dimensiones de sus componentes y su repartición en clases granulométricas. Las dimensiones de un grano es una noción difícil de definir, a causa de las formas variadas que se pueden encontrar (plaquetas, pajas, agujas, esferas). La forma y el tamaño de las partículas son conceptos implicados en la descripción de numerosos fenómenos de interés científico y tecnológico, entre estos cabe citar (Curso, 2000; Gómez et al., 1990):

- Ecuaciones de estimación de la cinética y consumo energético en procesos de adecuación morfológica, como molienda y sinterización; y establecimiento de criterios de la eficacia de clasificación en seco, como tamices, filtros, ciclones e impactadores.

- Modelos de distribución de fuerzas y tensiones en almacenamiento de gráneles, como apilamientos, silos y tolvas; y cálculos de flujo de gráneles a través de sistemas de vaciado y conducciones.

- Modelos de transmisión de calor y difusión molecular, particularmente lechos fijos y fluidizados.

- Modelos macroscópicos de reactividad química heterogénea y heterocatálisis.

Si bien en las universidades no se enseñan cursos específicos de tecnología de partículas, desde el punto de vista científico es una ciencia bastante desarrollada. En la Tabla 2 se presenta una lista general de los métodos de análisis de la tecnología de partículas disponibles en el mercado. 
Tabla 2. Métodos de análisis de la tecnología de partículas

\begin{tabular}{|c|c|}
\hline $\begin{array}{l}\text { Métodos de } \\
\text { microscopía: } \\
\text { - Óptica } \\
\text { - Microscopía electró- } \\
\text { nica de transmisión, } \\
\text { MET. } \\
\text { - Microscopía electró- } \\
\text { nica de barrido, MEB. } \\
\text { - Microscopía de fuerza } \\
\text { atómica, MFA. } \\
\text { - Análisis de imágenes. }\end{array}$ & $\begin{array}{l}\text { Métodos de propieda- } \\
\text { des eléctricas: } \\
\text { - Principio Coulter. } \\
\text { - Analizador de movili- } \\
\text { dad diferencial, } \\
\text { DMA. } \\
\text { - Movilidad electroforé- } \\
\text { tica. } \\
\text {-Potencial zeta. }\end{array}$ \\
\hline $\begin{array}{l}\text { Métodos de } \\
\text { interacción ligera: } \\
\text { - Difracción láser. } \\
\text { - Dispersión de luz de } \\
\text { una sola partícula. } \\
\text { - Dispersión de luz de } \\
\text { múltiples ángulos } \\
\text { - Oscurecimiento de luz } \\
\text { de una sola partícula. } \\
\text { - Doppler de fibra óp- } \\
\text { tica. } \\
\text { - Anemometría. }\end{array}$ & $\begin{array}{l}\text { Méfodos de } \\
\text { Clasificación: } \\
\text { - Clasificación de célu- } \\
\text { las activadas por } \\
\text { - Fluorescencia, FACS. } \\
\text { - Fraccionamiento de } \\
\text { flujo de campo, FFF. } \\
\text { - Clasificación de aire. } \\
\text { - Impactadores inercia- } \\
\text { les. }\end{array}$ \\
\hline $\begin{array}{l}\text { Método de } \\
\text { sedimentación: } \\
\text { - Foto sedimentación. } \\
\text { - Sedimentación centrí- } \\
\text { fuga } \\
\text { - Sedimentación de } \\
\text { rayos X. }\end{array}$ & $\begin{array}{l}\text { Métodos Mecánicos: } \\
\text { - Tamizado } \\
\text { - Ultrafiltración }\end{array}$ \\
\hline
\end{tabular}

La composición granulométrica tiene una gran importancia en la industria cerámica ya que de ella depende la superficie específica. Cuanto más fina sea la arcilla mayor resultará la superficie, más alto el porcentaje de humedad de moldeo y la contracción de secado y más angosto el diámetro de los capilares existentes en la pieza verde, lo cual dificultará el proceso de secado. Al reducirse la sección de los poros y aumentarse el número de contactos entre las partículas arcillo- sas se incrementará la resistencia mecánica en seco (Albella, 1999). En cocción, dado que el proceso de vitrificación comienza por la superficie de las partículas, cuanto mayor sea ésta más rápidamente y a más baja temperatura vitrificará, obteniéndose una pieza cocida de menor porosidad y mayor resistencia mecánica. Así, por ejemplo, en la Figura 2 se muestra una curva típica de distribución granulométrica de una arcilla caolinítica de la región sierra del Ecuador. La caolinita presenta tamaños de grano sensiblemente mayores que otras clases de arcillas (Guillen et al., 2012). Así, por ejemplo, orientativamente en una muestra, el porcentaje de partículas comprendidas entre 0,5 y 5 micras suele indicar el contenido en caolinita, mientras la fracción inferior a 0,5 micras representa el contenido en montmorillonita. Como consecuencia de esta granulometría más gruesa, las arcillas caoliníticas presentan una plasticidad algo baja, pero secan con rapidez y sin problemas a causa de su mayor porosidad en seco (Fernández, 2010). 


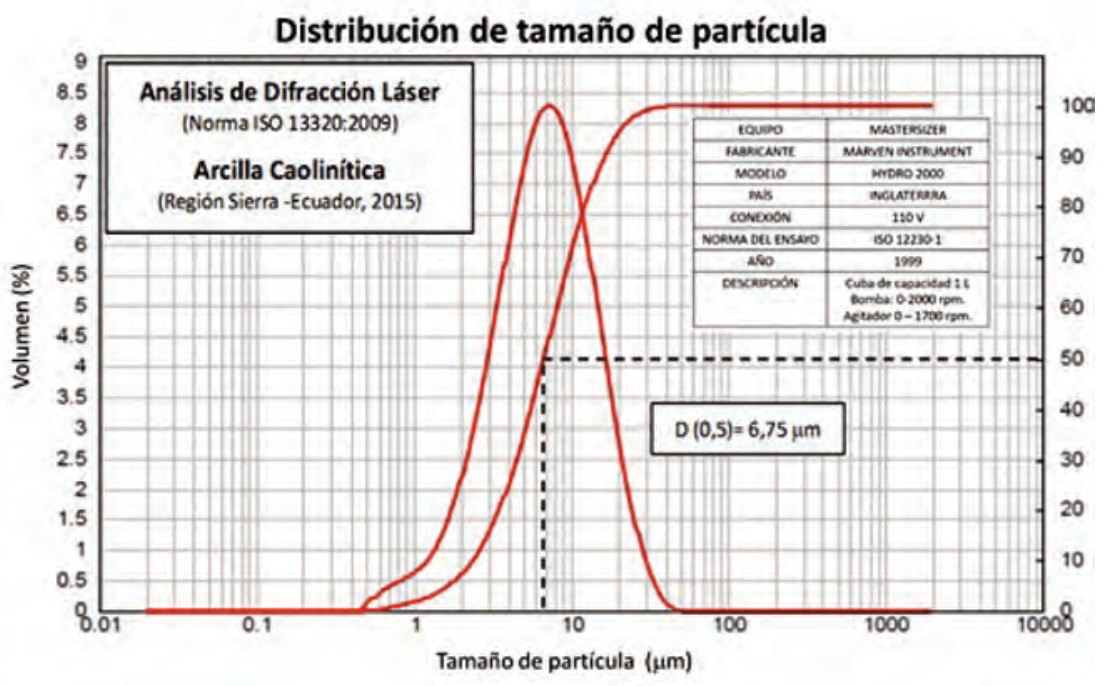

Figura 2. Curva de análisis granulométrico, difracción láser.

Arcilla caolinítica - Región Sierra. Ecuador

\section{Porosidad}

Los poros se suelen agrupar en tres grandes grupos, en función de su diámetro medio de acceso. Los macroporos $(\varnothing>50 \mathrm{~nm})$, mesoporos $(2<\varnothing<50 \mathrm{~nm})$ y microporos $(\varnothing$ $<2 \mathrm{~nm}$ ). Para la determinación del tamaño de poro se emplean por regla general las siguientes técnicas: (AlbeIla, 1999; Espinoza et al., 1978).

- Métodos basados en procesos de adsorción de gases, siendo de particular interés los basados en fenómenos de histéresis, los cuales están asociados a efectos de condensación capilar. Estos métodos son válidos para tamaños de poros entre $(2<\varnothing<20 \mathrm{~nm})$.

- Porosimetría de mercurio, que permite determinar poros entre $(10<\varnothing<150 \mathrm{~nm})$.

- Difracción de rayos-X a bajo ángulo, que da información sobre poros entre $(1<\varnothing<100 \mathrm{~nm})$.

La técnica más utilizada para determinar la porosidad abierta y la distribución de tamaños de poros abiertos en nuevos materiales y en especial los cerámicos, es la porosimetría de mercurio. Esta técnica determina el volumen de mercurio forzado a en- 
trar en los poros como una función de la presión, la cual está relacionada con el tamaño de poro mediante la ecuación de Washburn (Van Brakel et al., 1981):

$$
P=\frac{2 \cdot \gamma \cdot \cos \theta}{r}
$$

Donde P es la presión aplicada, $(\gamma)$ es la tensión superficial del mercurio medida a la temperatura del ensayo, ( $\theta)$ el ángulo de contacto entre el mercurio y las paredes del capilar o poro, y $(r)$ el radio equivalente del poro que se llena con mercurio.

Las muestras se colocan en un recipiente impermeable el cual es sometido a vacío y rellenado con un volumen conocido de mercurio, posteriormente se aplica presión al mercurio de forma progresiva. A baja presión el mercurio sólo es forzado a penetrar en canales porosos de gran diámetro de la muestra, así el cambio de volumen es pequeño. Cuando se aumenta la presión, el mercurio penetra en los poros más pequeños y hay una gran reducción de volumen.

En equipos como el porosímetro de mercurio modelo Autopore II 9220 de la casa Micromerics, el intervalo de medida de poros del equipo es aproximadamente entre $3 \mathrm{~nm}$ a 360 $\mathrm{mm}$ y el rango de presiones de intrusión de mercurio de $4 \times 10^{-3}$ a 414 MPa. Los ensayos se realizan utilizando distintas cantidades de muestra $(0,6-1,0$ g), comprobándose que la distribución de tamaños de poro no presenta dependencia con esta variable experimental. Por el contrario, la proporción relativa de porosidad abierta de las muestras, calculada a partir de la masa y de la cantidad total de porosidad abierta, presenta un incremento sistemático al aumentar la cantidad de muestra utilizada. Por ello, para contar con resultados comparables de este parámetro se utilizan iguales cantidades de muestra para todos los materiales.

En la Figura 3 se muestra la distribución de tamaños de poros, obtenida por porosimetría de mercurio, de compactos en verde de $\mathrm{Al}_{2} \mathrm{O}_{3}$, obtenidos por prensado isostático (A-P-v) y procesamiento coloidal (A-S-v), las curvas nos indican cuantitativamente diferencias en el empaquetamiento de las partículas, en estos compactos. Las distribuciones de poros son monomodales y estrechas para todos los compactos. Para los compactos obte- 
nidos a partir del procesamiento coloidal las distribuciones se encuentran desplazadas hacia tamaños inferiores; la diferencia, superior al $45 \%$, entre los valores del tamaño medio de poros de los compactos prensados isostáticamente y los obtenidos por procesamiento coloidal, evidencia este desplazamiento. Como han puesto de manifiesto diversos autores, los compactos en verde obtenidos a partir de un procesamiento coloidal alcanzan por lo general mayor eficiencia de empaquetamiento $y$, por consiguiente, mayor densidad que los obtenidos por procesamiento convencional (Murashov et al., 2011).

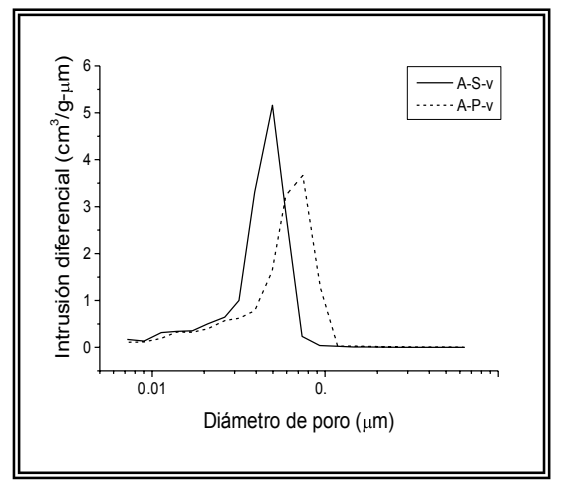

Figura 3. Efecto del procesamiento en la porosidad de compactos en verde de $\mathrm{Al}_{2} \mathrm{O}_{3}$, obtenidos por prensado isostático $(A-P-v)$ y procesamiento coloidal (A-S-v) (Uribe, 2001)
Evaluación de las propiedades mecánicas

La evaluación de las propiedades mecánicas para la caracterización de los materiales, en la que se incluyen los materiales compuestos, se hace fundamentalmente utilizando ensayos destructivos. El proceso de ensayo de materiales consiste en tomar determinada cantidad de muestras de un material y realizar cierto número de pruebas para caracterizar el comportamiento de este. Los ensayos mecánicos se realizan ante circunstancias simuladas y con equipo adecuado, de tal forma que los resultados tengan validez. Asimismo, existen normas para los procedimientos de caracterización mecánica. A nivel mundial, la normativa disponible es extensa. Es así como institutos normalizadores como ASTM, ASME, ISO, UNE, ICONTEC, INEN, entre otros, han generado gran cantidad de normas para diferentes propósitos, entre los que se incluye la caracterización mecánica.

La Sociedad Americana para Ensayos y Materiales, ASTM, ha desarrollado normas para ensayo de materiales entre las cuales se pueden encontrar las normas de caracterización de 
composites. De esta forma, los análisis para la caracterización mecánica de los materiales se fundamentan básicamente en los ensayos de compresión, tracción, flexión e impacto, bajo procedimientos ASTM. Así, por ejemplo, para la realización de estos ensayos, el Laboratorio de Análisis de Esfuerzos y Vibraciones "LAEV" de la Escuela Politécnica Nacional del Ecuador, cuenta con la máquina de ensayos universales Tinius Olsen modelo "Súper L", con capacidades de 132000 lbf (587165 N) y 130000 lbf (578269 N) y la máquina universal de ensayos Tinius Olsen modelo "H25KS", con una capacidad de 5620 lbf $(25000 \mathrm{~N})$.

\section{Ensayo de compresión}

El ensayo de compresión es uno de los ensayos mecánicos tensión-deformación más comúnmente realizado. El material ensayado se deforma hasta la rotura de la probeta, mediante la aplicación de una fuerza uniaxial a lo largo del eje principal que aumenta de manera gradual (Uribe, 2001). Por ejemplo, en cuanto a materiales cerámicos refractarios indica la factibilidad de uso del refractario en la construcción de hornos, dando el grado de adherencia entre granos y/o el sistema ligante. Las probetas para ensayar deben presentar dimensiones específicas indicadas en la norma mencionada en la Tabla 1, estas requieren mostrar superficies libres de defectos visibles, fisuras o imperfecciones. Las marcas ocasionadas por operaciones de mecanizado de la probeta serán cuidadosamente eliminadas con una lima fina o un abrasivo y las superficies limadas serán "suavizadas" con papel abrasivo. Así la resistencia a la compresión es la resistencia que presenta un cuerpo refractario antes de la aparición de la primera grieta, bajo el esfuerzo de compresión. Es importante considerar los factores que influyen en la resistencia mecánica a la compresión: densidad aparente, método de preparación, porosidad y temperatura de uso. Así una mayor proporción de porosidad implica mayor facilidad de generación de fallas, por ejemplo, un refractario cerámico moldeable puede alcanzar valores máximos de resistencia mecánica a la compresión de $300 \mathrm{~kg} / \mathrm{cm}^{2}$ a diferencia de un ladrillo denso de alúmina que puede alcanzar los 700 $\mathrm{kg} / \mathrm{cm}^{2}$ (Camillo, 2005). 


\section{Ensayo de flexión}

Este método de ensayo determina la rigidez a la flexión y las propiedades de resistencia mecánica de un mineral arcilloso o compuesto cerámico. El procedimiento aplicado corresponde a un sistema de carga en tres puntos utilizando una carga central en una viga con apoyos simples (ASTM C674). Este ensayo es realizado mediante la aplicación de la carga como lo indica la Figura 4, las propiedades a flexión pueden variar dependiendo de la superficie de la probeta, pues ningún laminado es perfectamente simétrico. Estas diferencias desviarán el eje neutral y los resultados se verán afectados incluso por la mínima asimetría presente en el laminado. Las propiedades a flexión también pueden variar de acuerdo con el espesor de la probeta, ambientes condicionantes o de ensayo en el que se ejecutan las pruebas, velocidad de aplicación de esfuerzo. Cuando se evalúan distintos materiales, los parámetros deben ser equivalentes para la comparación de todos los datos obtenidos.

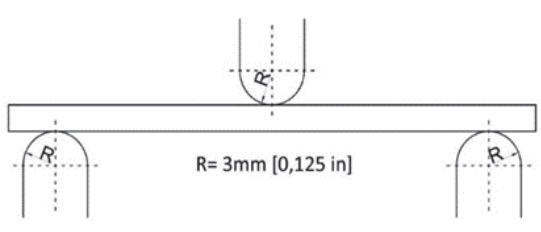

\section{Figura 4. Configuración de carga a flexión en tres puntos utilizada (ASTM C674)}

Para la realización de este ensayo, se requieren al menos cinco (5) probetas por cada condición de ensayo, a menos que se puedan obtener resultados válidos mediante el ensayo de menos probetas, como en el caso de un diseño experimental.

Las dimensiones de las probetas requeridas para los ensayos se encuentran indicadas en la norma ASTM, y de igual manera, las respectivas ecuaciones que permiten calcular valores para las propiedades a flexión analizadas. Al igual que en los ensayos de tracción y de impacto, las probetas deben respetar características solicitadas de aspecto superficial, como es la ausencia de fisuras o defectos que influyan erradamente en los resultados del ensayo (Cely et al., 2015). 


\section{Dureza. La indentación en materia- les frágiles}

La aplicación de la técnica de indentación ha proporcionado importantes avances en el estudio de la respuesta a la deformación y fractura de los materiales sometidos a esfuerzos de tensión. En comparación con los inconvenientes de las técnicas convencionales de determinación de la tenacidad (generalmente ensayos de flexión en 3 o 4 puntos en probetas entalladas), la técnica de indentación tiene una gran aceptación para evaluar el comportamiento relativo de materiales en proceso de investigación y desarrollo, ya que es una técnica simple, que no requiere grandes volúmenes de material ni costosos procesos de preparación de probetas (Uribe, 2001).

Cuando se hace presión con un cuerpo duro y rígido (penetrador) en la superficie lisa y plana de un material frágil, en la zona cercana al punto de aplicación de la carga el material sufre una deformación, inicialmente elástica y posteriormente plástica. AI retirar la carga permanece una impresión residual (huella) cuyas dimensiones son función de la dureza del material y de las características del penetrador. Si la presión aplicada es suficientemente alta, se llega a producir la fractura del material. La proporción relativa de deformación plástica y fractura viene determinada por la tenacidad del material.

La geometría de las zonas de deformación plástica y fractura depende de la geometría del penetrador. Estos se pueden clasificar en dos grupos, esféricos y afilados, en función de que su geometría sea esférica o piramidal, respectivamente. De los penetradores afilados, los más utilizados son los de tipo Vickers y Knoop, los cuales originan una geometría de impresión que no cambia según va a avanzando el penetrador en el material. La presión media de contacto resulta independiente del tamaño de la impresión y permite una medida adecuada de la dureza del material, $\mathrm{H}$. En el caso de una huella tipo Vickers la dureza, HV, viene dada por:

$$
H_{v}=\frac{\operatorname{sen} \varphi}{2} \cdot \frac{P}{a^{2}}
$$

Donde P es la carga aplicada, a es la semilongitud de la diagonal de la huella de indentación y $\varphi$ viene de $2 \varphi=136^{\circ}$ que corresponde al ángulo 
entre las caras del penetrador Vickers. En la Figura 5 se muestra el esquema de formación de las grietas cuando un penetrador Vickers penetra en un material. La secuencia del proceso de deformación y generación de grietas al aumentar la carga aplicada es la siguiente:

- En primer lugar, se forma una zona de deformación plástica (Figura 5a), cuyo tamaño aumenta al aumentar la carga. La relación carga/tamaño de la zona viene determinada por la dureza del material.

- Cuando la carga aplicada alcanza un valor crítico, se forma una grieta debajo del punto de contacto penetrador-material (Figura 5b). Se denomina grieta mediana y está situada en el plano de simetría del campo de tensiones creado.

- Para un tamaño crítico de la zona de deformación plástica, las grietas medianas se desestabilizan y se propagan hacia la superficie del material, formando dos grietas circulares bajo la superficie (Figura 5c).

- Al retirar la carga, las grietas "subsuperficiales" circulares se propagan hasta la superficie dando lugar a dos grietas semicirculares superficiales (Figura 5d) que emergen de los vértices de la huella, son mutua- mente perpendiculares y se sitúan en direcciones paralelas a las diagonales del penetrador (Figura 5f), estas grietas se denominan radiales.

En algunos materiales se forman antes las grietas radiales que las medianas (Figura 5e). Así debajo de la impronta Vickers se pueden encontrar dos geometrías de fisuras propagándose en los planos centrales debajo de la impronta: geometría Palmqvist (Figura 5e) y geometría radial (Figura 5f). Ambas configuraciones tienen un aspecto superficial similar y experimentalmente se ha observado que la primera configuración ocurre en materiales de alta tenacidad.

Es posible que se origine un sistema de grietas interiores adicionales denominadas grietas laterales, las cuales se forman en la discontinuidad zona plástica/material elástico, son paralelas a la superficie de la muestra $y$, cuando la muestra es severamente cargada, pueden girar interceptando la superficie. Estas grietas son las responsables de que, en algunas ocasiones, se observe el desprendimiento del material alrededor de la huella (desconchado, "chipping" en terminología anglosajona). 


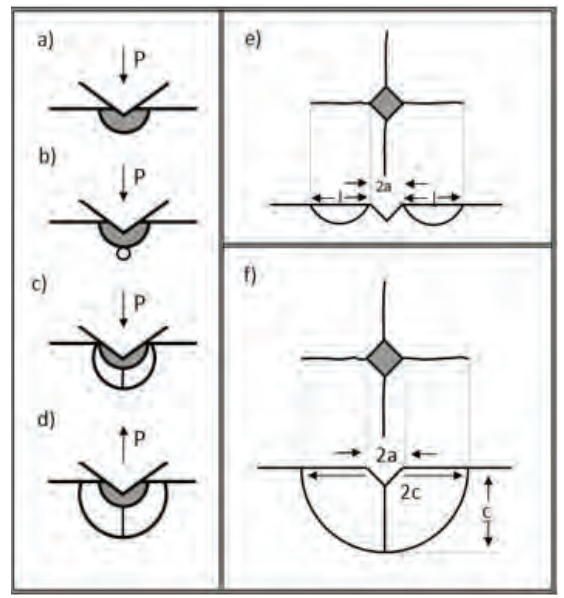

Figura 5. Esquema de formación de las grietas producida por un penetrador Vickers: a) zona de deformación plástica, b) formación de la grieta mediana, c) desarrollo de la grieta circular interna, d) Desarrollo de las grietas radiales. En $(e)$ y $(f)$ vista normal de la huella producida en la superficie: e) sección a largo de un diagonal tipo Palmqvist y f) Sección a lo largo de un diagonal tipo radial

Uno de los aspectos característicos del mecanismo de formación de grietas durante la indentación es el de que, en la mayoría de los casos, las grietas radiales $y / o$ laterales crecen hasta su tamaño final cuando el penetrador se retira. Esto ha obligado a admitir la existencia de una tensión residual, resultado de la incompatibilidad entre la zona deformada plásticamente y el material (elástico).
Las tensiones, que durante la carga son de tracción y tienden a abrir la grieta mediana, son de compresión durante la descarga. Cerca de la superficie el fenómeno es el inverso, durante la carga las tensiones son de compresión y durante la descarga se convierten en tensiones de tracción, haciendo que las grietas semicirculares, parcialmente desarrolladas, se propaguen completamente.

En la Figura 6 se muestra una imagen de la huella de indentación Vickers, realizada con una carga $\mathrm{P}=98 \mathrm{~N}$, en la superficie pulida de una muestra de $\mathrm{Al}_{2} \mathrm{O}_{3}-10 \% \mathrm{Al}_{2} \mathrm{TiO}_{5}$ (A-10AT). En la micrografía obtenida por microscopía óptica de luz reflejada, MOLR, se muestran los criterios de definición de una huella no válida: Grietas múltiples, huella de indentación asimétrica y grietas radiales no paralelas a las diagonales de la huella de indentación. 


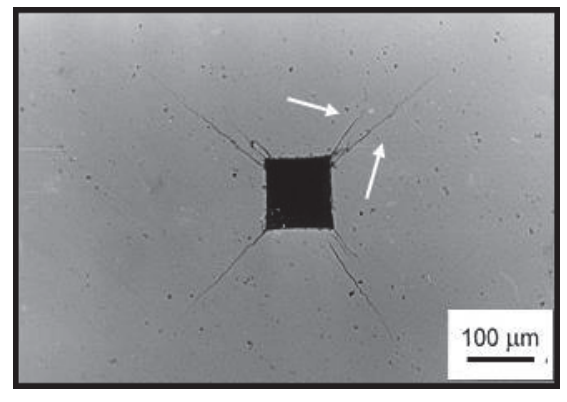

Figura 6. Imagen de la huella de indentación Vickers, carga $P=98 \mathrm{~N}$, en superficie pulida de una muestra de $\mathrm{Al}_{2} \mathrm{O}_{3}-10 \% \mathrm{Al}_{2} \mathrm{TiO}_{5}$ (Uribe, 2001)

Técnicas de análisis químico y mineralógico

El desarrollo gradual de la tecnología, en especial de las técnicas instrumentales de análisis, ha permitido obtener más información sobre los fenómenos que ocurren incluso a escala nanométrica. Los métodos de caracterización juegan un papel importante en los procesos de síntesis de nanopartículas cerámicas, ya que permiten determinar si se están obteniendo los resultados deseados en cuanto a forma, composición y distribución de tamaños. Varias técnicas de análisis han contribuido de forma eficaz al progreso y evolución de la nanotecnología; por ejemplo, para la determinación del tamaño de las partículas se puede emplear microsco- pía, dispersión de luz láser, difracción (Reventós et al., 2002).

Para garantizar una caracterización confiable de los materiales existen estándares desarrollados por comités técnicos de ciertas organizaciones, tales como la ASTM Internacional, Comisión Internacional Electrotécnica, Organización para la Cooperación Económica y Desarrollo (OECD), entre otros. Uno de estos comités es el ISO/TC229 WG4, encargado de realizar documentos guías y especificaciones para la caracterización de nanomateriales (Murashov et al., 2011). Un ejemplo de esto es la norma ISO/TS 10798:2011, que se refiere a la caracterización de nanotubos de carbono de pared simple utilizando microscopía electrónica de barrido y análisis de espectroscopia de rayos $X$. Cabe señalar que también existen normas de salud ocupacional y medioambiental, por ejemplo, la norma ISO/TR 13121: 2011 referente a la evaluación de riesgos de los nanomateriales (ISO/TS, 2011).

\section{Técnicas de análisis químico}

Las técnicas de análisis químico están basadas en la medida de la in- 
tensidad de radiación correspondiente a las transiciones electrónicas entre el estado fundamental y los estados excitados de los átomos. Cuando la transición se produce desde el estado fundamental hasta el estado excitado del átomo mediante la absorción de radiación de una determinada frecuencia (característica para cada átomo), estamos en el caso de las técnicas de absorción (Reventós et al., 2002). En el caso en que los átomos se lleven previamente a un estado excitado y se mide la intensidad de radiación emitida a la frecuencia característica correspondiente a la transición desde el estado excitado al estado fundamental, hablamos de técnicas espectrofotométricas de emisión (Albella et al., 1999).

Por ser todas ellas suficientemente conocidas no se describen sus fundamentos, recomendándose la extensa obra de Potts (1987) y Willard et al. (1991). Mundialmente el análisis químico de materiales y en especial de los polvos se realiza siguiendo la metodología y protocolos establecidos por el laboratorio de análisis. Los factores que se consideran a la hora de elegir la técnica analítica más ade- cuada son la precisión y la exactitud de los resultados. Por ejemplo, para el análisis químico de un mineral no metálico se pueden seleccionar las siguientes técnicas (Uribe, 2001):

- Gravimetría: para la determinación de la $\mathrm{SiO}_{2}$.

- Fotometría de llama: para la determinación de los óxidos alcalinos $\left(\mathrm{Na}_{2} \mathrm{O}, \mathrm{K}_{2} \mathrm{O}\right)$.

- Espectrometría de emisión atómica con fuente de plasma acoplado por inducción (ICP-AES): para la determinación del $\mathrm{Fe}_{2} \mathrm{O}_{3}, \mathrm{TiO}_{2}, \mathrm{Al}_{2} \mathrm{O}_{3}$, $\mathrm{CaO}, \mathrm{MgO}, \mathrm{SiO}_{2}$.

- La Fotometría de llama utiliza una Ilama como fuente de excitación y un fotodetector electrónico como dispositivo de medida. Se trata principalmente de un método sencillo y preciso de análisis cuantitativo, para determinación de metales alcalinos, la mayor parte de los alcalinotérreos y algún otro elemento metálico, aunque es posible analizar todas las longitudes de onda del espectro de emisión (espectrofotometría de Ilama o fotometría de llama).

- Espectrometría de Emisión Atómica con fuente de plasma acoplado por inducción ICP con detector de estado sólido, tiene la ventaja de 
poder captar simultáneamente todo el espectro, desde el ultravioleta hasta el infrarrojo gracias al detector de estado sólido. Con este tipo de detector se puede utilizar la práctica totalidad de líneas de emisión de cualquier elemento incluidas en la librería espectral. El equipo va provisto de un monocromador de red plana con dispersión cruzada que proporciona un gran poder de resolución y un espectro bidimensional.

En el caso del estudio de los minerales no metálicos y en los especifico los arcillosos la presencia o ausencia de determinados elementos en determinadas proporciones, puede, en efecto, tener significado en la determinación del comportamiento a la cocción, más, sin embargo, pocas veces proporciona información alguna sobre la trabajabilidad de la materia en estudio (molienda, atomización, prensado, etc.). Lo interesante es poder combinar los datos compositivos con los datos estructurales (ver apartado de análisis térmicos), a fin de obtener resultados precisos y óptimos del comportamiento tecnológico del mineral o composición en estudio (Sánchez et al., 1978).
En la Tabla 3 se puede observar que las materias primas objeto de estudio poseen tenores de óxidos del orden establecido para minerales arcillosos, lo que permite inferir un buen comportamiento de las arcillas en producción. Sin embargo, no se puede limitar este comportamiento a la lista de óxidos presentes, ya que estos pueden combinarse de distinto modo para dar lugar a fases mineralógicas de diversa y significativa importancia de comportamiento en la producción, sin dejar de considerar, por otra parte, la variabilidad de composición en el mismo yacimiento arcilloso. Sin embargo, podemos acotar positivamente que la proporción de óxidos férrico detectado es muy inferior al límite máximo (10\%) permitido en productos de quema roja. Asimismo, otros óxidos $\left(\mathrm{Na}_{2} \mathrm{O}, \mathrm{K}_{2} \mathrm{O}, \mathrm{TiO}_{2}\right.$, etc.), están presentes en cantidades mínimas y globalmente no superan el $5 \%$ máximo permitido. Los productos cerámicos también adquieren diferentes coloraciones en función de la relación de los diferentes óxidos presentes en las arcillas, por ejemplo, se obtiene el color amarillo, marrón claro y oscuro cuando se da la relación $\mathrm{Fe}_{2} \mathrm{O}_{3}: \mathrm{CaO}=0,6: 0$, 8 y da color amarillo brillante y amarillo, si 
$\mathrm{Fe}_{2} \mathrm{O}_{3}: \mathrm{CaO}=0,5: 0,6$. Los óxidos de manganeso proporcionan el color naranja y el color negro (Uribe, 2020).

Tabla 3. Análisis químico por ICP de minerales arcillosos de la Sierra de Ecuador, según la norma ASTM C323 (Uribe, 2020)

\begin{tabular}{lcccccccccc}
\hline $\begin{array}{l}\text { Mineral Arcilloso } \\
\text { Análisis Químico (\%) }\end{array}$ & $\mathrm{SiO}_{2}$ & $\mathrm{Al}_{2} \mathrm{O}_{3}$ & $\mathrm{Fe}_{2} \mathrm{O}_{3}$ & $\mathrm{CaO}$ & $\mathrm{MgO}$ & $\mathrm{Na}_{2} \mathrm{O}$ & $\mathrm{K}_{2} \mathrm{O}$ & $\mathrm{TiO}_{2}$ & P.F. & Total \\
\hline Arcilla (AR-PI-15-01) & 66,24 & 17,25 & 4,23 & 0,99 & 0,75 & 1,62 & 0,84 & 0,55 & 7,53 & 100 \\
\hline Arcilla (AR-BO-15-01) & 60,12 & 20,04 & 3,33 & 2,04 & 0,95 & 1,89 & 0,83 & 0,53 & 10,27 & 100 \\
\hline Arenosa (SI-CH-15-01) & 75,33 & 7,56 & 3,66 & 2,97 & 0,31 & 1,99 & 1,81 & 0,33 & 6,01 & 100 \\
\hline Arena (SI-PI-15-01) & 89,37 & 1,01 & 4,54 & 0,05 & 0,22 & 0,30 & 0,14 & 0,09 & 4,28 & 100 \\
\hline
\end{tabular}

\section{Difracción de rayos $X$}

Los rayos $\mathrm{X}$ son una forma de radiación electromagnética de elevada energía. La longitud de onda de estos rayos está en el orden de $10^{-10} \mathrm{~m}$ y es similar a la distancia interatómica que existe en los materiales cristalinos, (Cañada, 2006). La difracción de rayos $\mathrm{X}$ es un fenómeno de interferencias constructivas, que consiste en la incidencia de un haz de rayos $X$ sobre un material sólido. Para que se realice este proceso se debe cumplir con la ley de Bragg y los átomos del material sólido deben tener una disposición ordenada dentro de su estructura. La ley de Bragg relaciona la longitud de onda de los rayos $\mathrm{X}$ con la distancia interatómica y el ángulo de incidencia del haz sobre la muestra (Melgarejo et al., 2010).

La difracción de rayos $X$ permite la identificación de fases cristalinas de la muestra, el análisis cualitativo y cuantitativo de muestras cristalinas, policristalinas y amorfas y puede ser aplicada en los siguientes campos:

- Química inorgánica, cristalografía, física del estado sólido, física aplicada, mineralogía, química analítica, química orgánica, farmacología.

- Ciencia de materiales: cerámicos, materiales de la construcción, catálisis.

- Ciencias ambientales: residuos só- 
lidos cristalinos, polvos en suspensión.

- Arqueología: análisis de fases de muestras.

Específicamente, en el área de la nanotecnología cerámica, esta técnica permite determinar la cristalinidad y el parámetro de red de nanopartículas, nanocables y películas delgadas (Cao, 2004), así como también la estequiometria de las nanopartículas sintetizadas, con lo cual es posible determinar si se han obtenido los resultados esperados de la síntesis y si ha existido alguna contaminación en la muestra.

En las Figuras 7 y 8 se muestran algunos ejemplos de determinación de naturaleza mineralógica en minerales No metálicos y de evolución de fases cristalinas en materiales estructurales, mediante difracción de rayos $X$.

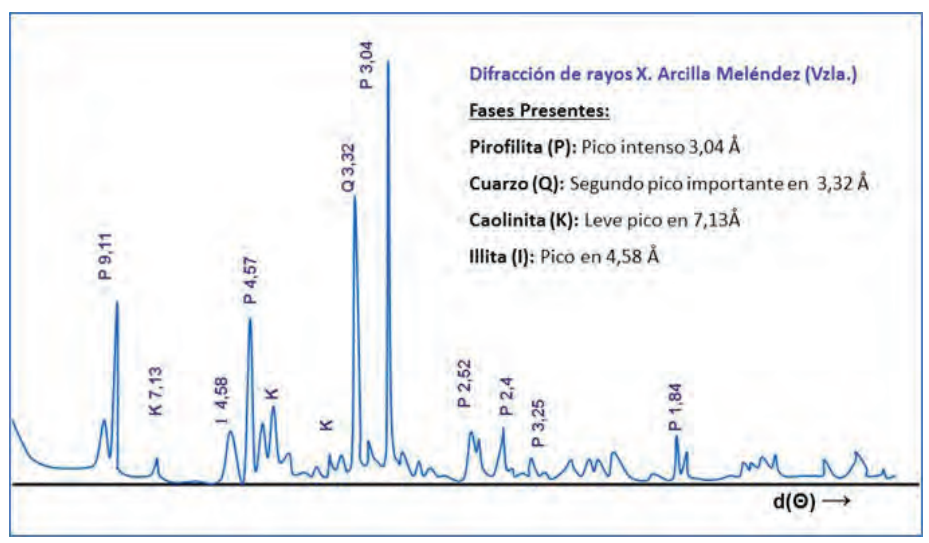

Figura 7. Difractogramas de rayos X de polvos. Arcilla Meléndez, Venezuela 


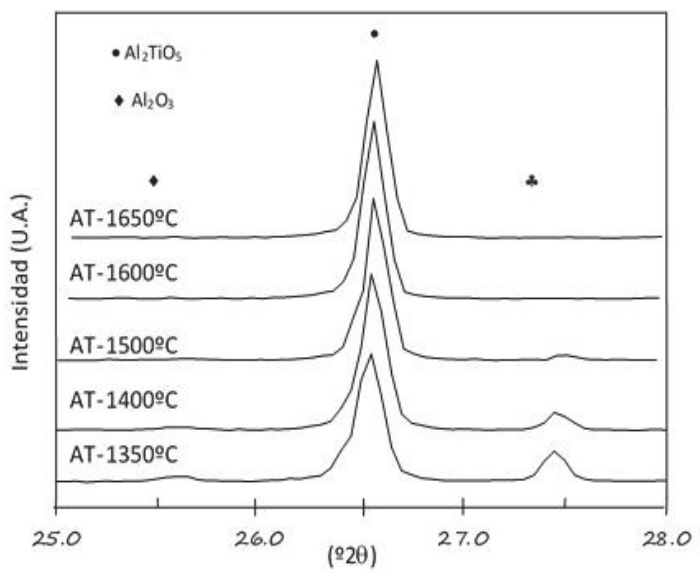

Figura 8. Difractogramas de rayos $\mathrm{X}$ de polvos. Materiales de $\mathrm{Al}_{2} \mathrm{TiO}_{5}$ obtenidos a partir de un ciclo térmico simple,

a las temperaturas indicadas y durante 3 horas (Uribe, 2001)

\section{Microscopía electrónica}

Los microscopios electrónicos son instrumentos científicos que, en lugar de utilizar luz visible, utilizan un haz de electrones de alta energía para examinar y caracterizar objetos en escala nanométrica. El haz de electrones posee una longitud de onda de alrededor de $0,5 \AA$, que permite observar objetos en el orden manométrico (Martín, 2020). En microscopía electrónica se requiere que los electrones sean acelerados mediante altos voltajes y que el sistema trabaje con presiones muy bajas, incluso en el vacío, para evitar que los electrones que viajan con una trayectoria prefijada, desde la fuente hasta la muestra, se desvíen por la presencia de átomos o moléculas extrañas que no son parte de la muestra a analizar (Torres et al., 2011).

Existen dos tipos de microscopios principalmente, el microscopio electrónico de barrido y el microscopio electrónico de transmisión. Ambos utilizan a los electrones como una fuente de iluminación, lentes electromagnéticas para que converjan el haz de electrones sobre la muestra, lentes objetivos para que se pueda magnificar la imagen y lentes protectores que permiten observar la forma, 
la estructura interna y el ordenamiento atómico de la muestra. Con estos microscopios se puede combinar un detector de rayos $\mathrm{X}$ para determinar la composición atómica de la muestra que se está observando (Martín, 2020).

\section{Microscopía electrónica de barrido, MEB}

Es importante mencionar que la muestra a ser analizada mediante microscopía electrónica de barrido debe cumplir ciertos requerimientos, tales como ser compatible con el sistema de vacío del instrumento y ser eléctricamente conductora. Las muestras que no son conductoras pueden ser recubiertas con una fina capa conductora $(5 \mathrm{~nm})$ de un metal como el oro, platino, paladio, etc. (Martín, 2020). Las ventajas que presenta el microscopio electrónico de barrido son (Poblete, 2020):

- Utiliza un haz móvil de electrones que escanea punto por punto la muestra en áreas seleccionadas, por lo que se obtiene una imagen ampliada tridimensional y realista de la superficie del objeto.

- La muestra no requiere el corte en capas para ser observada.
En la caracterización de nanopartículas, el MEB provee información sobre el tamaño de partícula, morfología, presencia de aglomerados. Además, al combinarse este método con capacidades de análisis químico, el microscopio electrónico de barrido es capaz de proporcionar información detallada de la composición química (Poblete, 2020). En la Figura 9, se muestra una imagen de un compuesto de estructural de $\mathrm{Al}_{2} \mathrm{O}_{3}-10 \%$ $\mathrm{Al}_{2} \mathrm{TiO}_{5}$, obtenido vía prensado isostático $(\mathrm{P})$ y vía suspensión $(\mathrm{S})$, sometidos a un proceso de sinterización controlada a $1500{ }^{\circ} \mathrm{C}$. Se observa el efecto del procesamiento en el crecimiento de los granos de la matriz de $\mathrm{Al}_{2} \mathrm{O}_{3}$, factor que se evidencia cuantitativamente con la curva de distribución de tamaño de partícula obtenida a partir de la técnica de análisis de imagen.

\section{Espectroscopia de infrarrojos}

La espectroscopía es una técnica de análisis que estudia la interacción de la radiación electromagnética con la materia y mide la luz absorbida a diferentes longitudes de onda. La espectroscopía de infrarrojo se basa en la absorción de la radiación infrarroja por las moléculas en vibración, su 


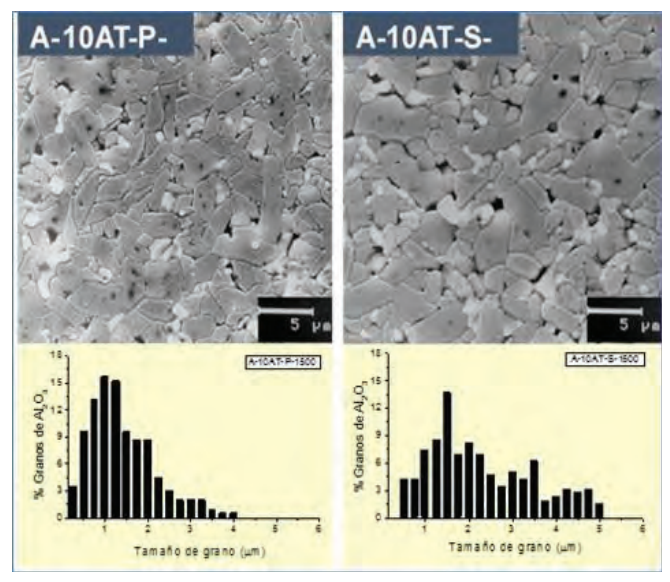

Figura 9. Imagen de compuestos de estructurales de $\mathrm{Al}_{2} \mathrm{O}_{3}-(10 \%) \mathrm{Al}_{2} \mathrm{TiO}_{5}$ obtenidos vía prensado isostático (P) y vía suspensión (S) (Uribe, 2001)

objetivo principal es la determinación de los grupos funcionales presentes en una muestra, la cual puede ser sólida, líquida o gaseosa. Una molécula absorberá la energía de un haz de luz infrarroja cuando la energía incidente sea igual a la necesaria para que se dé un cambio de vibración en la molécula, este tipo de vibraciones deben generar un cambio en el momento dipolar de la molécula para que se produzca la absorción (Skoog et al., 2001).
La energía relacionada con el infrarrojo no es lo suficientemente alta como para excitar los electrones, pero puede inducir excitación vibratoria de los átomos unidos por enlaces covalentes. Las vibraciones que experimentan los átomos están en el rango de 1011 a 1013 Hz. En la Figura 10 se puede observar un espectro obtenido para una muestra de Hidroxiapatita, en el cual se puede apreciar los diferentes grupos funcionales encontrados en la muestra (Uvillus, 2018). 


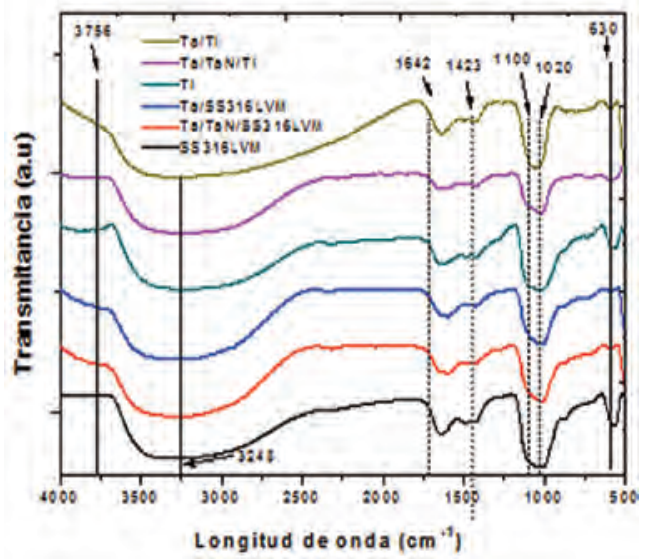

Figura 10. Análisis FTIR de la deposición de Hidroxiapatita a las mejores condiciones encontradas en el método electroquímico, en los diferentes sustratos estudiados

(Uvillus, 2018)

La espectroscopía de infrarrojo es ampliamente usada en la caracterización de compuestos, el requisito más importante para utilizar esta técnica es que la molécula a analizar sea activa en la región del IR. Entre sus ventajas podemos citar las siguientes: Caracterización de compuestos orgánicos e inorgánicos, análisis de muestras en estado sólido, líquido o gaseoso, identificación de grupos funcionales en sustancias desconocidas, análisis cuantitativo de las muestras y análisis de films poliméricos.

\section{Técnicas de análisis térmico}

Cuando algún parámetro físico de un material es medido de manera conti- nua, en función del aumento y/o variación controlada de la temperatura a la que está siendo sometido, podemos decir que estamos en presencia de un análisis térmico. Esta denominación engloba un conjunto de técnicas analíticas que estudian el comportamiento térmico de los materiales. Cuando un material se calienta o se enfría, su estado, estructura cristalina y su composición química pueden sufrir cambios más o menos importantes (Camillo, 2005):

- Fusión: paso del estado sólido al estado líquido.

- Sublimación: paso del estado sólido al estado gaseoso.

- Solidificación: paso del estado lí- 
quido al estado sólido.

- Cristalización: paso al estado sólido ordenado.

- Amorfización: paso al estado sólido desordenado.

- Transición: cambio en su estructura cristalina.

- Reacciones: de oxidación, alteración, descomposición, etc.

- Expansión y compresiones en su volumen.

- Cambios texturales: sinterización, recristalización, etc.

En el análisis térmico:

- La Termogravimetría / Análisis Termogravimétrico, ATG (Thermo Gravimetric Analysis, TGA): configura la base de los cambios de perdida y/o ganancia de peso del material sometido a tratamiento.

- El Análisis Térmico Diferencial, ATD, (Differential Thermal Analysis, DTA) o la Calorimetría Diferencial de Barrido (Differential Scanning Calorimetry, DSC): configura la medida de los cambios de energía, es decir indica si una reacción es endotérmica o exotérmica, incluso se puede medir la variación de calor.

- El Análisis Termomecánico, TMA: (Thermomechanical Analysis, ATM): mide la variación dimensional del cuerpo en función de la temperatura, y en algunos casos en simultáneo con la aplicación de una carga.

Este tipo de análisis, además de representar un obvio método para observar y prever el comportamiento de una materia prima o de una composición (pasta cerámica), durante el secado, cocción o el enfriamiento, representan una óptima ayuda para la determinación de la composición mineralógica de una mezcla, al observar los diferentes tipos de efectos en estrecha correlación con la estructura cristalina y las transformaciones de fase de los diferentes minerales, y representan también una posible ayuda en la determinación de los parámetros químicos, por ejemplo mediante la posibilidad de reconocer la presencia de minerales y sales como la dolomita, carbonatos, sulfatos, fluoruros, etc. (SACMI-ATC, 2004).

Los instrumentos para los análisis térmicos se componen fundamentalmente de un cabezal de medida, apropiado para acoger la muestra a analizar en la justa forma física y transformar las variaciones de esta 
muestra en señales eléctricas amplifícales y manejables, y de un sistema de calentamiento, basado normalmente en resistencias eléctricas, que debe presentar fuertes características de estabilidad, homogeneidad y programabilidad, asegurando así la perfecta reproducibilidad de las medidas (SACMI-ATC, 2004).

Durante el calentamiento de un mineral se pueden presentar cambios físicos y químicos (Camillo, 2005, Cely et al., 2015):

- Los cambios físicos están relacionados con las transiciones polimorfas, por ejemplo, del $\alpha$-cuarzo trigonal al $\beta$-cuarzo hexagonal y con la fusión de minerales.

- Los cambios químicos son más diversificados durante el calentamiento mineral, los más comunes se pueden observar en la Tabla 4:

Tabla 4. Cambios químicos asociados al calentamiento de minerales arcillosos

\begin{tabular}{ll}
\hline Disociación & $\mathrm{Ca}\left(\mathrm{CO}_{3}\right) \rightarrow \mathrm{CaO}+\mathrm{CO}_{2}$ \\
\hline Deshidratación & $\begin{array}{l}\mathrm{Ca}\left(\mathrm{SO}_{4}\right)_{2} \mathrm{H}_{2} \mathrm{O} \rightarrow \\
\mathrm{Ca}\left(\mathrm{SO}_{4}\right)+2 \mathrm{H}_{2} \mathrm{O}\end{array}$ \\
\hline Descomposición & $\mathrm{Fe}\left(\mathrm{CO}_{3}\right) \rightarrow \mathrm{FeO}+\mathrm{CO}_{2}$ \\
\hline $\begin{array}{l}\text { Descomposición } \\
\text { e intercambio }\end{array}$ & $\mathrm{Ca}\left(\mathrm{CO}_{3}\right)+\mathrm{SiO}_{2} \rightarrow$ \\
\hline
\end{tabular}

- Los mismos procesos en minerales diferentes con estructuras del mismo tipo se caracterizan por temperaturas distintas, debido a las diferencias en la composición.

- Las impurezas químicas también influyen en las temperaturas de los cambios en los minerales.

- Aparte de la temperatura, cada proceso se caracteriza por su "calor interno". Algunas reacciones producen calor y tienen lugar espontáneamente, una vez alcanzada la temperatura necesaria, son reacciones exotérmicas. En otros casos, el mineral calentado necesita un aporte adicional de calor para empezar y desarrollar la reacción, son reacciones endotérmicas.

- Otra característica importante de las alteraciones térmicas en minerales es el cambio de peso, relacionado con la pérdida de los componentes volátiles $\mathrm{CO}_{2}$ y $\mathrm{H}_{2} \mathrm{O}$.

\section{Análisis termogravimétrico, ATG}

Está basado en la medida de la variación de la masa de una muestra cuando esta se somete a un cambio de temperatura en una atmósfera controlada. Esta variación puede ser una pérdida o una ganancia de masa. El registro de estos cambios nos dará 
información sobre si la muestra se descompone o reacciona con otros componentes. La Termogravimetría puede utilizarse conjuntamente con otras técnicas, como por ejemplo ATD o DSC, ya que permiten obtener información complementaria sobre el comportamiento térmico de una muestra. En el ATG se estudian los siguientes tipos de reacciones (Donat, 2005):

- Sólidos que se descomponen formando gases

- Sólidos que se descomponen formando un residuo sólido con uno o varios gases

- Sólidos que reaccionan sobre un gas y formando un residuo gaseoso

- Reacción de dos sólidos con formación de un nuevo sólido y de un gas por lo menos

- Formación de un gas sólido sobre un gas con formación de un nuevo sólido y de uno o varios gases

En cuanto a los factores que afectan la forma de lo termogramas tenemos (Donat, 2005):

- La naturaleza de la atmósfera a la cual se realiza el ensayo

- La forma del crisol

- La disposición del material dentro del crisol
- El estado de división de la materia

- La masa de materia utilizada

- La velocidad de calentamiento

- El estado higrométrico de la materia

En las curvas del ATG obtenidas de minerales arcillosos se observan pérdidas de peso provocadas por:

- Pérdidas de agua

- Descomposición de los hidratos

- Descomposición de los carbonatos

- Descomposición de la materia orgánica

- Oxidación, sulfatación, carburación (producen aumento de peso)

\section{Análisis térmico diferencial, ATD}

Se someten a un ciclo térmico tanto a la muestra como a un material de referencia, que es inerte desde el punto de vista térmico, físico y químico, y se mide la diferencia de temperatura entre la muestra y el material de referencia, en función del tiempo (temperatura constante) o de la temperatura alcanzada en cada momento. En principio, se trata de una técnica cualitativa que permite detectar si se dan procesos endotérmicos o exotérmicos en nuestra muestra, e indica la temperatura a la cual tienen lugar estos cambios energéticos. Con 
un adecuado calibrado del equipo es posible convertir el ATD en una técnica semicuantitativa para poder obtener información sobre la cantidad de calor involucrado en los procesos. En los métodos de ATD o DSC los cambios que experimenta el material se controlan midiendo la diferencia de temperatura $(T)$ entre una muestra (S) y una de referencia (R) para el ATD y, midiendo para mantener a la misma temperatura (S) y (R) la cantidad de calor que hay que suministrar para mantener el equilibrio, cuando se trabaja el DSC. Este registro de flujo de calor suministra una medida de la cantidad de energía absorbida o desprendida en una determinada transición (Albella, 1999). Factores que afectan las curvas ATD/DSC debidos a la propia muestra:

- Cantidad de muestra (área de pico proporcional)

- Tamaño de partícula (polvos, laminas)

- Compactación de la muestra (interacción atmosfera del horno)

- Capacidad calorífica y conductividad térmica (forma y grado de compactación)

- Diluyentes (sin reacción con la muestra)
Factores instrumentales que afectan las curvas ATD/DSC:

- Velocidad de calentamiento (de 5 a $20 \mathrm{C} / \mathrm{min}$ aumenta temperatura y pico de reacción)

- Naturaleza del portamuestras (vidrio, cuarzo, cerámico, metálico y aluminio para DSC)

- Termopares ( en contacto directo con portamuestras)

- Atmósfera del horno (estática o dinámica, activa o inerte)

Así para optimizar los resultados del ATD/DSC esencialmente debemos considerar (Albella, 1999):

- Cantidad de muestra: La elección de la cantidad de muestra depende de la naturaleza del problema a investigar. Si queremos medir un efecto térmico de muy pequeña magnitud, tales como las transiciones de segundo orden o transiciones vítreas en polímeros; será necesario utilizar más muestra que si queremos medir un efecto térmico muy acusado, tal como un punto de fusión. Una buena cantidad de muestra suele ser entre 1-10 mg. Trabajando una pequeña cantidad de muestra se consigue:

- Máxima resolución de picos - Picos en forma regular 
- Mejor contacto con el portamuestras

- Facilita la eliminación de volátiles

- Minimiza el gradiente térmico en la muestra

- Permite utilizar mayores velocidades de calentamiento.

Por otra parte, trabajar con grandes cantidades de muestra:

- Permite la detección de pequeños efectos endotérmicos

- Las medidas de las cantidades son más precisas

- Se produce mayor cantidad de productos volátiles.
Velocidad de calentamiento: Si se trabaja con grandes cantidades de muestra es necesario trabajar a velocidades bajas, debido a mayores gradientes de temperatura.

\section{Curvas características ATG y ATD}

En la imagen de la Figura 11 se observan las reacciones endotérmicas y exotérmicas típicas del ATD, asimismo la curva de variación de masa del ATG. Se observa que por lo general estas curvas se presentan simultáneamente en una misma imagen, a fin de verificar la coincidencia los distintos procesos térmicos que sufre el material, cuando es sometido a ciclos de sinterización controlados.
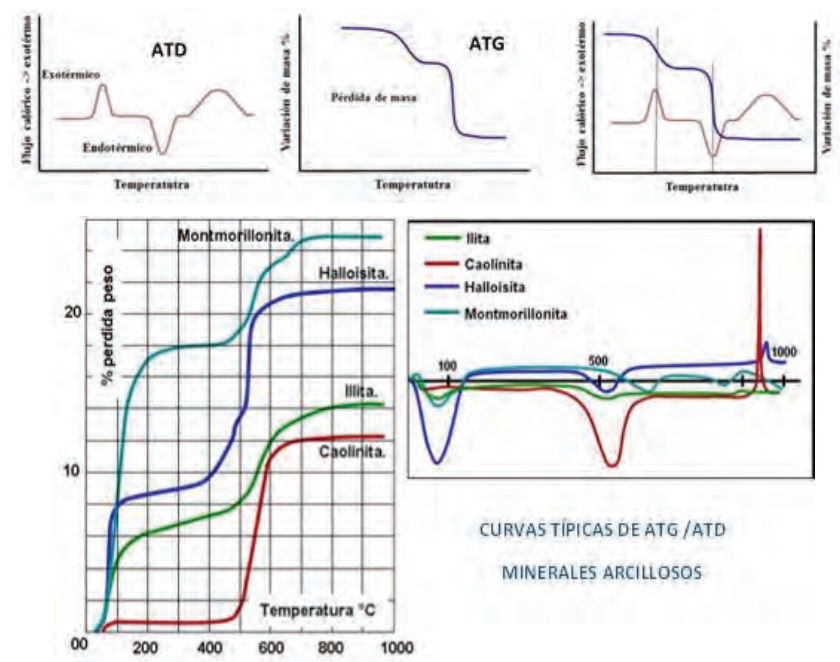

CURVAS TIPPICAS DE ATG/ATD

MINERALES ARCILLOSOS

Figura 11. Curvas típicas del Análisis Térmico Diferencial, ATD y Análisis Térmico Gravimétrico, ATG de minerales arcillosos 
En la imagen de la Figura 12 se observan las reacciones endotérmicas y exotérmicas típicas del ATD y ATG de una arcilla venezolana. En la curva ATD se observa:

- A 91,59 ${ }^{\circ} \mathrm{C}$, se produce una reacción endotérmica. Dicha reacción es debida a la salida del agua de humedad en el material.

- A $313,91{ }^{\circ} \mathrm{C}$ un leve pico endotérmico es observado, debido a la pérdida de hidratos presentes Goetita $\left(\mathrm{Fe}_{2} \mathrm{O}_{3} \cdot 3 \mathrm{H}_{2} \mathrm{O}\right)$ y Halloisita: $\left(2 \mathrm{SiO}_{2} \cdot \mathrm{AL}_{2} \mathrm{O}_{3} \cdot \mathrm{nH}_{2} \mathrm{O}\right)$. La presencia de los hidratos también fue observada mediante en los espectros de rayos $X$.

- A 525,52 ${ }^{\circ} \mathrm{C}$, se aprecia una fuerte reacción endotérmica producto de la eliminación del agua de constitución de la caolinita.

- A 571,06 ${ }^{\circ} \mathrm{C}$, ocurre la transformación alotrópica del cuarzo $\alpha \rightarrow$ cuarzo $ß$, dando como resultado un pico endotérmico cuya intensi- dad dependerá de la cantidad de cuarzo en la muestra.

- Se observa un pico exotérmico aproximadamente sobre los $1000{ }^{\circ} \mathrm{C}$, causado por la formación de muIlita; $2 \mathrm{SIO}_{2} 3 \mathrm{AL}_{2} \mathrm{O}_{3}$.

En la curva ATG se observa:

- Entre la temperatura ambiente 25$110{ }^{\circ} \mathrm{C}$, se produce una primera pérdida de peso de aproximadamente $0,2 \%$, la cual es producto de la eliminación del agua de humedad contenida en el material.

- La presencia de hidratos de hierro y Halloisita generaron en el material una segunda pérdida de masa, del orden del $1 \%$ entre los 130 $350{ }^{\circ} \mathrm{C}$.

- En el intervalo de temperatura de 450-590 ${ }^{\circ} \mathrm{C}$, se observa una pérdida de masa de aproximadamente un $3,7 \%$, causada por la eliminación del agua de constitución. 

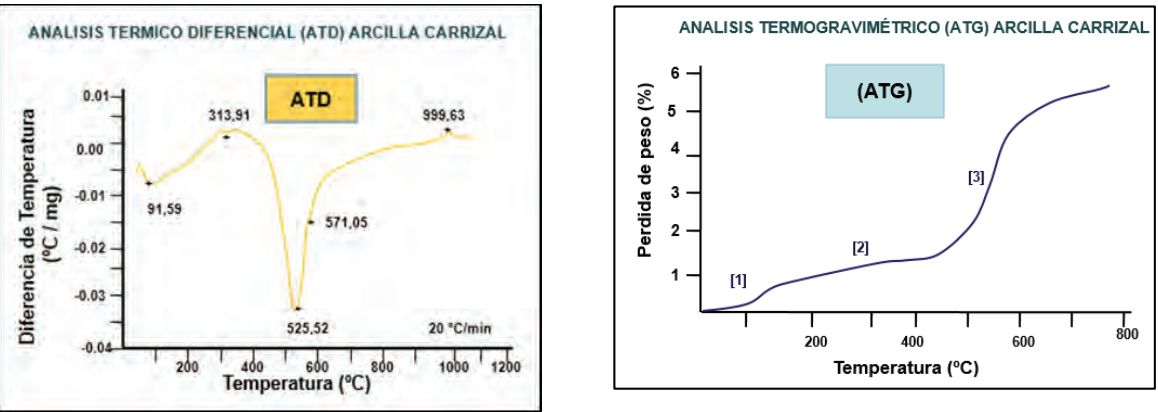

Figura 12. Curvas típicas del Análisis Térmico Diferencial, ATD y Análisis Térmico Gravimétrico, ATG de una arcilla venezolana

\section{CONCLUSIÓN}

Gracias a que se ha dado paso a la normalización mundial de métodos, ensayos y prácticas de análisis, acompañados éstos de equipos de medición de alta tecnología, de gran precisión y exactitud, el aporte científico actual está dado por la posibilidad no solo de caracterizar los materiales cerámicos, sino también de evaluar, predecir y diseñar el comportamiento de los nuevos materiales y lo más importante valorar su utilidad en diversas aplicaciones tanto industriales como artesanales. En función de esto se hace necesaria la valorización especializada descrita de materias primas, procesos y materiales cerámicos, a fin de alcanzar el control optimo y sistematizado, tanto de las características de los minerales arcillosos, como de las propiedades de los productos cerámicos.

\section{AGRADECIMIENTOS}

Los autores agradecen el aporte investigativo realizado por el Centro de Asistencia a la Industria Cerámica perteneciente a FUNDATEC, Caracas Venezuela. Asimismo, agradecen al Vicerrectorado de Investigación y Pro- yección Social de la Escuela Politécnica Nacional por el apoyo y financiamiento prestado a través del proyecto PIS-16-13: "Optimización tecnológica de productos cerámicos industriales y/o artesanales del Ecuador". 


\section{LISTA DE REFERENCIAS}

Albella J. M. (1999), Introducción a la ciencia de los materiales. CSIC. Madrid, España.

Belmonte M., González J., Miranzo P. y Osendi M. (2010). Material cerámico de nitruro de silicio con función gradiente continua in situ, procedimiento de elaboración, propiedades y sus aplicaciones. Oficina Española de Patentes y Marcas. $\mathrm{P}^{\circ}$ de la Castellana, 75-28071 Madrid.

Cañada P. (2006). "Difracción de rayos X". http://www.uma.es/scai/servicios/xrd/xrd. html.

Cao G. (2004). "Nanostructures \& Nanomaterials Synthesis, Properties \& Applications". Londres: Imperial College Press.

Camillo F. (2005). Curso de Ciencias Cerámicas. Instituto Universitario de Tecnología Dr. Federico Rivero Palacio, FUNDATEC-CAIC. Caracas-Venezuela.

Cely L. y Bolívar R. (2015). "Materia prima para la industria cerámica de Norte de Santander. II. Evaluación del comportamiento térmico y su incidencia en las propiedades tecnológicas", Respuestas, 20(1) 84-94.

Curso de Postgrado: "Tecnología de Partículas y Análisis Granulométrico" (2000). INCAR-CSIC. Oviedo, España.

Díaz L. y Torrecillas R. (2002). Arcillas cerámicas: una revisión de sus distintos tipos, significados y aplicaciones. Bol. Soc. Esp. Cerám. Vidrio, 41(5) 459-470.

Espinoza de los Monteros J. (1979). Características y aplicaciones de materias primas cerámicas. Bol. Soc. Esp. Cerám. Vidrio, 18(4) 243-249.

Donat M. y Uribe R. (2005). Curso de Ciencias Cerámicas. Instituto Universitario de Tecnología Dr. Federico Rivero Palacio, FUNDATEC-CAIC. Caracas-Venezuela.

Fernández J.F. y Frutos J. (2003), Introducción a la Electrocerámica. Madrid, Fundación Rogelio Segovia para el desarrollo de las telecomunicaciones, Ciudad Universitaria. Programa CYTED Red VIII-F. 
Fernández M. (2010). Manual sobre fabricación de baldosas, tejas y ladrillos. Barcelona, España.

García A. (1985). Origen y composición de las arcillas cerámicas. Bol. Soc. Esp. Cerám. Vidrio, 24(6) 395-404.

Guillen J., Bribiesca S. y Escudero R. (2012). Caracterización y evaluación de un depósito de caolín de "Los Azufres" Michoacán, México, para su uso industrial. Bol. Soc. Esp. Cerám. Vidrio, 51(6), 329-336.

Gómez A., García G. y Sánchez P. (1990). Propiedades cerámicas de arcillas procedentes de Guadix (Granada). Bol. Soc. Esp. Cerám. Vidrio, 29(1), 37-40.

Hevia R. (2012). Materias primas: importancia de su conocimiento para la formulación cerámica. Cerámica y Cristal, 145, 48-52.

ISO International Organization of Standardization (2011). http://www.iso.org/iso/iso_catalogue/catalogue_tc/catalogue_detail.htm?csnumber $=46128$

Dávila J., Galeas S., Guerrero V., Pontón P., Rosas N., Sotomayor V. y Valdivieso C. (2011). Nuevos Materiales: Aplicaciones Estructurales e Industriales. Quito: Imprefepp.

Kingery W.D. (1976). Introduction to Ceramics. Jhon Wiley \& Sons, 2da edición.

Martín. J. (agosto, 2020) "Nanociencia y Nanotecnología, entre la Ciencia Ficción del Presente y la Tecnología del Futuro". http://www.oei.es/cienciayuniversidad/ spip.php?article193

Martín P. (1977). Tecnología de las arcillas. Bol. Soc. Esp. Cerám. Vidrio, 16(5), 295-300.

Melgarejo J.C., Proenza J.A., Galí S. y Llovet X. (2010). Técnicas de caracterización mineral y su aplicación en exploración y explotación minera. Boletín de la Sociedad Geológica Mexicana. 62(1), 1-23.

Murashov V. y Howard J. (2011). Nanotechnology Standards. New York, Estados Unidos: Springer. 
Paetsch D. (1977) Sobre el control de las materias primas cerámicas. Bol. Soc. Esp. Cerám. Vidrio, 17(3), 151-161.

Pinto E. A. (2011). Los materiales cerámicos. Editorial Alsina. Buenos Aires, Argentina. Poblete V. and Pilleux M. (2020) "Microscopía Electrónica de Barrido". [Online]. http://www.genealog.cl/cursos/id42a/Trabajos/15SEM/15SEM.doc

Reventós M., Clausell J., Esteve V., Delgado M., Ochando L., Ruíz J., Martí F. y Amigo J. (2002). Caracterización mineralógica de materias primas cerámicas por métodos cuantitativos de difracción de rayos X. Bol. Soc. Esp. Cerám. Vidrio, 41(6), 509-512.

SACMI-ATC (2004). Tecnología Cerámica Aplicada. Castellón- España.

Sánchez J., Sánchez I., Rasines I. y Iniguez J. (1978). Arcillas Cerámicas de Navarra. Bol. Soc. Esp. Cerám. Vidrio, 17(4) 209-217.

Sánchez P. y Rodríguez J. (1998). Características generales, propiedades, yacimientos y aplicaciones de pirofilita. I: Estructura, síntesis y características térmicas. Bol. Soc. Esp. Cerám. Vidrio, 37(4), 285-289.

Sánchez P. y Rodríguez J. (1998). Características generales, propiedades, yacimientos y aplicaciones de pirofilita. II. Yacimientos, aplicaciones y utilización como materia prima cerámica. Bol. Soc. Esp. Cerám. Vidrio, 37(5), 359-368.

Souza G., Sánchez R. y Holanda J. (2002). Characteristics and physical-mechanical properties of fired kaolinitic materials. Cerámica 48(306), 102-107.

Skoog D., Holler F. and Nieman T. (2001) "Principios de Análisis Instrumental". Madrid, España: McGraw-Hill, 2001.

Torres J., Gutiérrez R., Castelló R. y Vizcayno C. (2011). Análisis comparativo de caolines de diferentes fuentes para la producción de metacaolín. Revista Latinoamericana de Metalurgia y Materiales 31(1), 35-43.

Uribe R. (2001) Tesis Doctoral: Desarrollo de Materiales Estructurales de Alúmina -Titanato de Aluminio con Alta Resistencia al Choque Térmico. UAM, Madrid España. 
Uribe R. (2015). Investigaciones de materias primas minerales no metálicas en el Ecuador. Revista Politécnica, 36(1), 34-44.

Uribe R., Chico A., Rosas N., Lascano L. (2020). Minerales arcillosos del Ecuador. Protocolo de categorización cerámica: una revisión. InfoANALÍTICA - PUCE, 8(1); DOI: 10.26807/ia.v8i1.125

Van Brakel J., Modry S., and Svata M. (1981). "Mercury porosimetry," State of the Art. Powd.Techn. 29, 1-12.

Villalba Hervás (2020). Tecnología Industrial I. Materiales de uso técnico. http://apren demostecnologia.org/2010/01/18/tecnologia-industrial-i-materiales-de-uso-tecnico/ 